\title{
MicroRNA-365 inhibits proliferation, migration and invasion of glioma by targeting PIK3R3
}

\author{
YONGGANG ZHU ${ }^{1}$, HONGGUANG ZHAO ${ }^{2}$, MIN RAO $^{3}$ and SONGBAI XU ${ }^{4}$ \\ ${ }^{1}$ Department of Radiotherapy, China-Japan Union Hospital of Jilin University, Changchun, Jilin 130033; \\ ${ }^{2}$ Department of Nuclear Medicine, The First Hospital of Jilin University; ${ }^{3}$ Department of Gastroenterology, \\ The People's Republic Hospital of Jilin Province; ${ }^{4}$ Department of Neurosurgery, The First Hospital \\ of Jilin University, Changchun, Jilin 130021, P.R. China
}

Received September 6, 2016; Accepted February 1, 2017

DOI: $10.3892 /$ or.2017.5458

\begin{abstract}
A growing body of evidence suggests that microRNA-365 (miR-365) played crucial role in the initiation and development of many types of cancers. However, the biological role of miR-365 in human glioma remains unclear. Herein, the aims of this study were to investigate the role and underlying mechanisms of miR-365 in glioma by a series of in vitro and in vivo experiments. We found that miR-365 was strongly downregulated in malignant glioma tissues and cell lines. Restoration of the expression of miR-365 in glioma cells significantly inhibited cell proliferation, migration and invasion in vitro and tumor growth in vivo. Notably, phosphoinositide3-kinase regulatory subunit 3 (PIK3R3) was proved to be a direct target of miR-365 in glioma cells, and its mRNA expression was inversely correlated with miR-365 expression in clinical glioma tissues. PIK3R3 overexpression in miR-365 expressing cells could rescue proliferation, migration and invasion inhibition of miR-365. In addition, miR-365 was able to inhibit the phosphorylation of AKT and mTOR in vitro and in vivo, which are key participants in the AKT/mTOR pathway. These results suggest that miR-365 functioned as a tumor suppressor in glioma by targeting PIK3R3, suggesting that miR-365 has potential as therapeutic targets for glioma.
\end{abstract}

\section{Introduction}

Glioma is the most common tumor type in the central nervous system with high morbidity and mortality (1). Despite aggressive treatment including glioma surgery, radiotherapy, chemotherapy, gene therapy, immunotherapy and other novel biological therapies, the median survival duration of patients with advanced glioma has not significantly improved due to

Correspondence to: Dr Songbai Xu, Department of Neurosurgery, The First Hospital of Jilin University, 71 Xinming Street, Chaoyang, Changchun, Jilin 130021, P.R. China

E-mail: xusongbai616@sina.com

Key words: glioma, miR-365, proliferation, invasion, PIK3R3 its high recurrence and metastasis rate $(2,3)$. Therefore, an improved understanding of the biological basis of glioma progression might provide useful information for the clinical management of this disease.

MicroRNAs (miRNAs) are small, approximately 22 nucleotides in length, non-coding RNAs that negatively regulate gene expression at a post-translational level by binding to complementary sequences in the 3'UTRs of targeted mRNAs $(4,5)$. miRNAs have been reported to be involved in a range of cellular functions such as differentiation, proliferation, and apoptosis $(6,7)$. Accumulating evidence supports critical roles of miRNAs in the progression of different cancers, where they play a crucial role in tumor development through regulation of cellular proliferation, invasion, metastasis and apoptosis (8-10). Many miRNAs have been demonstrated to function as oncogenes or tumor suppressors in glioma $(11,12)$, which highlight the implications of miRNAs in diagnosis, treatment, and prognosis of glioma.

miR-365, a newly discovered miRNA, has been reported to be involved in tumor progression and development in several types of human cancers, such as lung cancer $(13,14)$, melanoma (15), gastric carcinoma (16), osteosarcoma (17), and colon cancer (18). However, the detail biological function and underlying molecular mechanism of miR-365 in glioma remains unclear. Therefore, the goals of the present study were to investigate the biological function and underlying molecular mechanism of miR-365 on the carcinogenesis of glioma. Our results showed that miR-365 expression was significantly downregulated in glioma cell lines and tissues. We also found that miR-365 overexpression inhibited cell proliferation, migration and invasion of glioma by targeting PIK3R3. Our results might contribute to the understanding of the molecular mechanism underlying glioma pathogenesis.

\section{Materials and methods}

Ethics statement. This study was approved by the Medical Ethics Committee of Jilin University (Changchun, China). All participating patients provided written informed consent for the use of surgical samples before surgery. All animals were treated in accordance with standard guidelines for the care and use of laboratory animals of Jilin University. 
Clinical samples and cell lines. Thirty-six pairs of glioma tissues and their adjacent normal brain tissues were collected from patients undergoing surgery at the first of Hospital of Jilin University from July 2012 to December 2014. All the tissues were snap-frozen in liquid nitrogen immediately after resection and stored at $-80^{\circ} \mathrm{C}$ until use.

Primary normal human astrocytes (NHA) and four human glioma cell lines (U251, U87, U118 and LN18) were from the Type Culture Collection of the Chinese Academy of Sciences (Shanghai, China). The cells were routinely cultured in Dulbecco's modified Eagle's medium (DMEM, Gibco, Grand Island, NY, USA) supplemented with $10 \%$ fetal bovine serum (FBS, Gibco), $100 \mathrm{U} / \mathrm{ml}$ penicillin and $100 \mathrm{mg} / \mathrm{ml}$ streptomycin (Sigma, St. Louis, $\mathrm{MO}$, USA) at $37^{\circ} \mathrm{C}$ with $5 \% \mathrm{CO}_{2}$ in a humidified atmosphere.

Isolation of RNA and quantitative real-time PCR (qRT-PCR). Total RNA was extracted from the glioma cell lines $\left(2 \times 10^{6} \mathrm{cells}\right)$ and glioma tissues $(100 \mathrm{mg}$ ) with TRIzol reagent (Invitrogen, Carlsbad, CA, USA) according to the manufacturer's protocol. The optical density of the RNA samples at $260 \mathrm{nM}$ was quantified by a NanoDrop ND-2000 Spectrophotometer (NanoDrop Technologies, Houston, TX, USA). For identification of miR-365 expression, complementary DNA (cDNA) were synthesized using TaqMan MicroRNA Reverse Transcription kit (Applied Biosystems, Foster City, CA, USA). qRT-PCR was performed with the TaqMan MicroRNA Assay for miR-365 and U6 (Ambion, Austin, TX, USA) and TaqMan Universal Master Mix II without UNG (Ambion) under ABI PRISM 7900 Sequence Detection System (Applied Biosystems). For determination of $P I K 3 R 3$ mRNA expression, reverse transcription (RT) were performed with a M-MLV First Strand kit (Invitrogen) using Oligo(dT) 20 primers (Invitrogen). qRT-PCR was performed using SYBR Select Master Mix (Invitrogen) under ABI PRISM 7900 Sequence Detection System. The primes of PIK3R3 and GAPDH used in this study were according to a previous study (19). The level of mature miR-365 was normalized relative to U6 endogenous control and $P I K 3 R 3$ mRNA expression was normalized relative to $G A P D H$ (endogenous control) using the $2^{-\Delta \Delta \mathrm{Ct}}$ method.

Cell transfection. miR-365 mimic and corresponding negative control miRNA (miR-NC) were purchased from Shanghai GenePharma (Shanghai, China). PIK3R3 overexpression plasmid (pCDNA3.1) was a gift of Dr Peng Zhang (Jilin University). Transfection was performed using Lipofectamine RNAiMAX (Invitrogen) according to the manufacturer's instructions.

Proliferation assay. Cell proliferation assay was performed using 3-(4,5-dimethyl-2-thiazolyl)-2,5-diphenyl-2H-tetrazolium bromide (MTT) (Sigma). Briefly, approx. 2000 transfected cells were seeded into each well of 96-well plates and cultured for 1-4 days. At the indicated time (24, 48, 72 and $96 \mathrm{~h}), 100 \mu \mathrm{l}$ fresh medium containing MTT $0.5 \mathrm{mg} / \mathrm{ml}$ was added into each well and cultured for $4 \mathrm{~h}$ at $37^{\circ} \mathrm{C}$, then the medium was replaced with $100 \mu 1$ of dimethyl sulfoxide (DMSO, Sigma) and shaken at room temperature for $10 \mathrm{~min}$. The absorption was measured at $490 \mathrm{~nm}$ with a microplate reader (Thermo Labsystems, Helsinki, Finland).
Invasion and migration and assays. The invasive ability of glioma cells was determined using 24-well Transwell chambers coated with Matrigel (BD Biosciences, San Jose, CA, USA). In brief, $1 \times 10^{5}$ transfected cells in serum-free medium were seeded at in the top chamber coated with Matrigel and incubated at $37^{\circ} \mathrm{C}$ in a humidified incubator containing 5\% $\mathrm{CO}_{2}$. The bottom chamber was filled with medium containing $10 \% \mathrm{FBS}$ as a chemoattractant. After $24 \mathrm{~h}$ of incubation, the non-invaded cells on the upper surface of the membrane were removed with a cotton swab, cells that invaded to the underside of the membrane were fixed with $70 \%$ ethanol for $30 \mathrm{~min}$ and stained with $0.2 \%$ crystal violet for $10 \mathrm{~min}$. Photographs were imaged, and the number was counted in five randomly selected fields under a light microscope (Olympus, Tokyo, Japan). For Transwell migration assays, glioma cells were determined using Transwell chambers without the Matrigel coating.

Dual luciferase reporter assay. The wild-type 3'-UTR segment of PIK3R3, which contained a putative binding site for miR-365, was amplified from normal human genomic DNA by PCR and inserted downstream of the luciferase gene in pGL3-control vector (Promega, Madison, WI, USA), named as Wt-PIK3R3. A mutant 3-UTR of PIK3R3 contained a mutation in the complementary seed region of miR-365 was amplified by PCR and inserted downstream of the luciferase gene in pGL3control vector, referred to as Mut-PIK3R3. U87 cells $\left(1 \times 10^{5}\right)$ were seeded in 24-well plates and grown to $60-70 \%$ confluence. Cells were then cotransfected with $200 \mathrm{ng}$ Wt-PIK3R3 or Mut-PIK3R3 reporter plasmid, $50 \mathrm{nmol} \mathrm{miR-365} \mathrm{mimic} \mathrm{or}$ miR-NC, and 20 ng pRL-TK Renilla plasmid (Promega) using Lipofectamine 2000 (Invitrogen). At $48 \mathrm{~h}$ after transfection, both firefly and Renilla luciferase activities were measured $48 \mathrm{~h}$ after transfection by using the Dual-Luciferase Reporter Assay System (Promega).

Animal studies. Five-week-old female BALB/c nude mice were purchased from the Animal Center of Jilin University (Changchun, China). All animal experiments were performed in accordance with the NIH Guide for the Care and Use of Laboratory Animals. U87 cells stable expressing miR-365 or miR-NC were injected subcutaneously into each side of the posterior flank of the nude mouse (six per group, $2 \times 10^{6}$ cells for each mouse). Tumor growth was examined every five day after injection, and tumor volumes were calculated using the equation $\mathrm{V}=\mathrm{AxB} / 2\left(\mathrm{~mm}^{3}\right)$, where $\mathrm{A}$ is the largest diameter and $\mathrm{B}$ is the perpendicular diameter. Five weeks after the implantation, the mice were sacrificed, and the xenograft tumors were excised, and weighted. Part of tumor tissues were stored for further analysis.

Western blotting. Western blot analysis was performed as previously described (20). Briefly, cultured cells or tissues were harvested and lysed in ice-cold RIPA buffer (Beyotime, Jiangsu, China) according to the manufacturer's instructions. The total concentrations of protein were determined using the BCA Protein assay kit (Beyotime). Equal amounts of proteins $(30 \mu \mathrm{g})$ were separated by $10 \%$ sodium dodecylsulfatepolyacrylamide gels (SDS-PAGE, Pierce, Rockford, IL, USA) and transferred onto nitrocellulose membranes (Millipore, Madison, WI, USA). After blocking with 5\% non-fat dry 
A

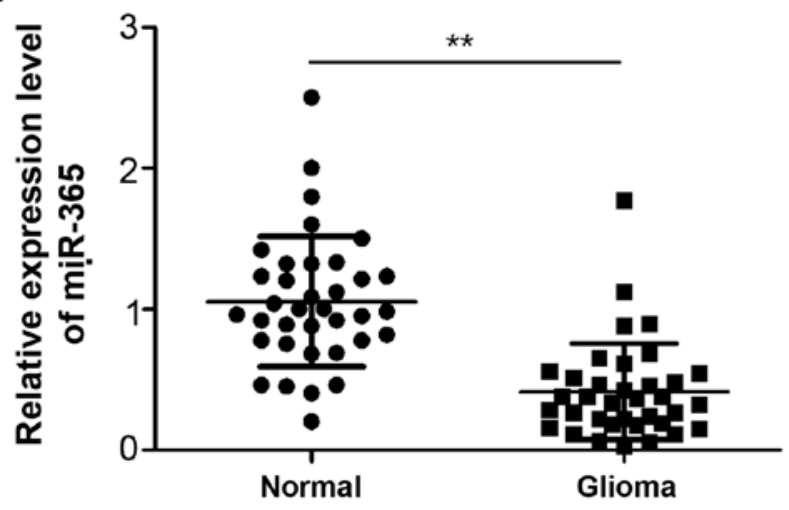

B

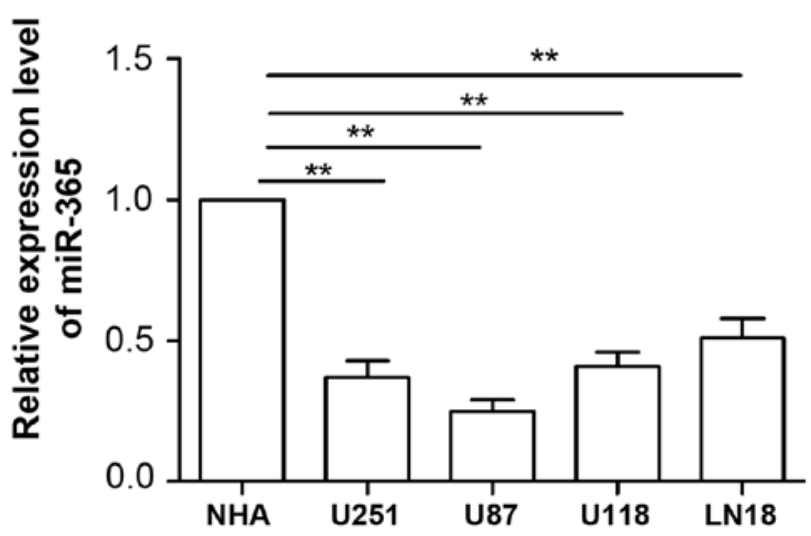

Figure 1. miR-365 was decreased in glioma tissues and cell lines. (A) The expression of miR-365 was determined in four human glioma cell lines (U251, U87, U118 and LN18) and normal human astrocytes (NHA). (B) The expression of miR-365 was determined in 36 paired glioma tissues and adjacent normal tissues by qRT-PCR. ${ }^{* *} \mathrm{P}<0.01$.

milk in TBST (20 mM Tris-HCl, $\mathrm{pH} 7.5,150 \mathrm{mM} \mathrm{NaCl}, 0.1 \%$ Tween-20), the membrane was incubated with mouse antihuman PIK3R3 monoclonal antibody (1:1000; Santa Cruz Biotechnology Inc., Santa Cruz, CA, USA), mouse anti-human AKT monoclonal antibody (1:1000; Santa Cruz Biotechnology Inc.), mouse anti-human p-AKT monoclonal antibody (1:1000; Santa Cruz Biotechnology Inc.), mouse anti-human mTOR monoclonal antibody (1:1000; Santa Cruz Biotechnology Inc.), mouse anti-human p-mTOR monoclonal antibody $(1: 1000$; Santa Cruz Biotechnology Inc.) and mouse anti-human GAPDH monoclonal antibody (1:5000; Santa Cruz Biotechnology Inc.) at $4^{\circ} \mathrm{C}$ overnight. The membrane was incubated with the goat anti-mouse IgG conjugated to horseradish peroxidase antibody (1:5000; Santa Cruz Biotechnology Inc.) at room temperature for $2 \mathrm{~h}$. The protein bland was observed using a chemiluminescent detection system (ECL, Thermo Scientific, Rockford, IL, USA) and exposed to X-ray film (Thermo Fisher Scientific).

Statistical analysis. All data are presented as the mean $\pm \mathrm{SD}$ (standard deviation) from at least three independent experiments. The SPSS software package (version 18.0, SPSS Inc.; Chicago, IL, USA) was used to perform the statistical analysis. A two tailed Student's t-test was used to evaluate the significance of differences between two groups. ANOVA was employed to analyze the significance of differences in more than two groups. The relationship between miR-365 and PIK3R3 expressions was tested using Spearman's correlation analysis. The significance level was set as $\mathrm{P}<0.05$.

\section{Results}

miR-365 is downregulated in human glioma cell lines and tissues. To examine levels of miR-365 in glioma, we first measured the expression of miR-365 in four human glioma cell lines (U251, U87, U118 and LN18) and normal human astrocytes (NHA) by qRT-PCR. As shown in Fig. 1A, the expression of miR-365 was markedly downregulated in all four human HCC cell lines U251, U87, U118 and LN18 compared with the NHA cell line. We also evaluated miR-365 expression in 36 glioma tissues and adjacent normal tissues. Consistent with the results from cell lines, miR-365 levels were significantly decreased in glioma tissues compared with normal tissues (Fig. 1B). These results indicated that miR-365 was downregulated in glioma.

miR-365 inhibits glioma cell proliferation, migration and invasion. To explore the possible biological functions of miR-365 in glioma, we transfected miR-365 mimic or miR-NC into U87 cells, which has lower expression of miR-365 (Fig. 1A), then transfection efficiency were determined by qRT-PCR. The results showed that miR-365 expression was higher in U87 cells transfected with miR-365 mimic compared to cells transfected with miR-NC (Fig. 2A). To demonstrate the effect of miR-365 on glioma growth, MTT assay was performed in glioma cells. As shown in Fig. 2B, overexpression of miR-365 in U87 attenuated cell proliferation from 48 to $96 \mathrm{~h}$ after transfection (Fig. 2B). We also investigated the effect of miR-365 overexpression on the migration and invasion abilities of glioma cells by Transwell assay. We found that miR-365 overexpression significantly decreased the migration and invasion capacity of U87 cells compared to miR-NC group (Fig. 2C and D). These results demonstrated that miR-365 suppressed glioma cell proliferation, migration and invasion.

PIK3R3 is a target of miR-365 in glioma cells. To understand how miR-365 suppresses glioma growth, migration and invasion, bioinformatics (miRTarBase and TargetScan) were used to identify the target of miR-365. PIK3R3 was chosen as a target of miR-365, since it has a binding sequence for miR-365 at position (67-73) of 3'UTR (Fig. 3A). To verify PIK3R3 as a direct target of miR-363, luciferase activity assay was performed. The results showed that miR-365 significantly inhibited the luciferase activity of wild-type 3'-UTR of PIK3R3 in U87 cells, but luciferase activity in mutant-type 3'-UTR of PIK3R3 in U87 cells was unchanged (Fig. 3B). Moreover, we found that overexpression of miR-365 significantly suppressed PIK3R3 expression on mRNA and protein levels (Fig. 3C and D). These results indicated that PIK3R3 is a direct target of miR-365 in glioma cells.

PIK3R3 expression was upregulated and inversely correlated with miR-365 expression in glioma tissues. Further experiments were performed to investigate the expression of PIK3R3 in glioma tissues and adjacent normal tissues by qRT-PCR. The result showed that the PIK3R3 mRNA expression was 
A

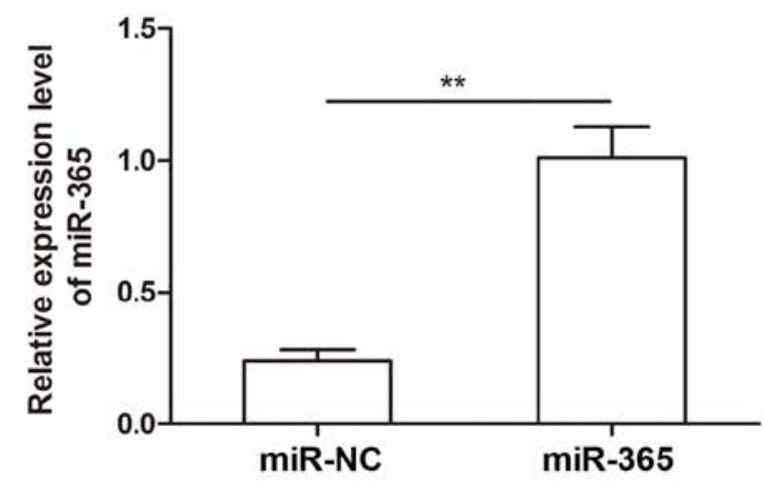

C

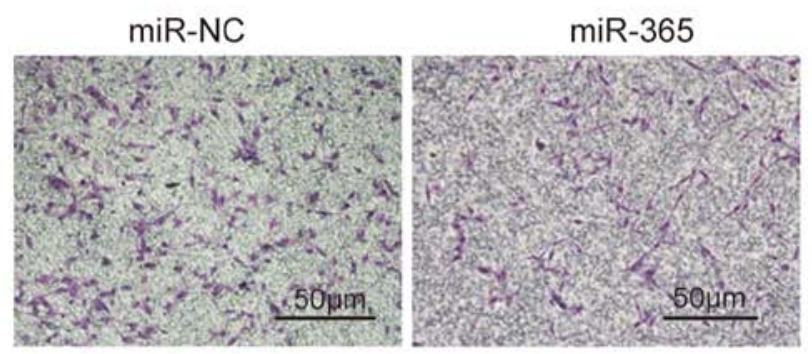

D

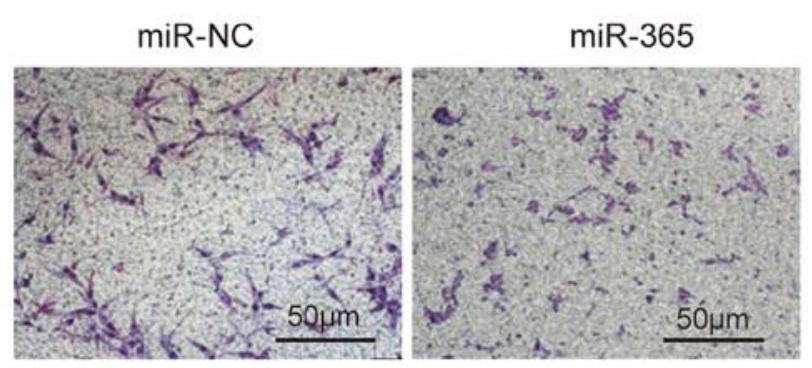

B
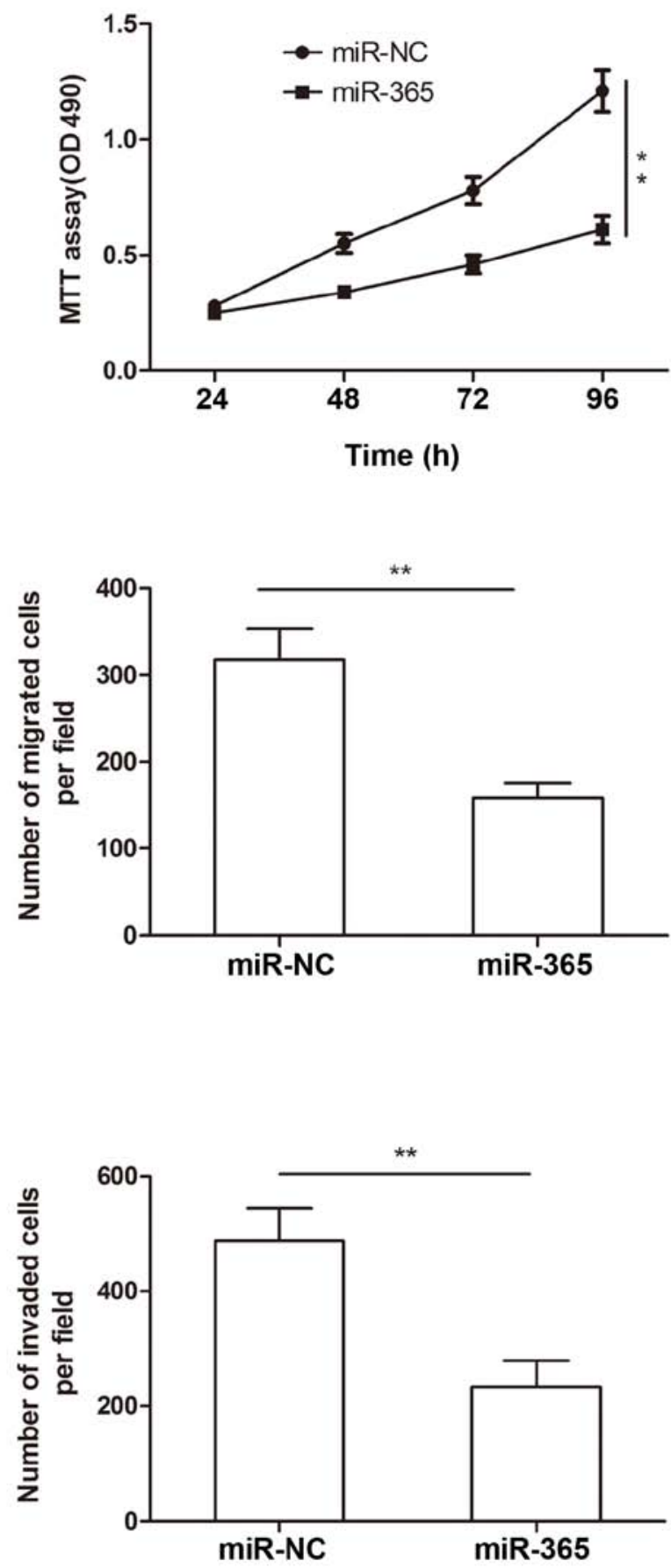

Figure 2. miR-365 inhibits glioma cell proliferation, migration and invasion. (A) The expression of miR-365 in U87 cells transfected with miR-365 mimic or miR-NC were detected by qRT-PCR. (B-D) Cell proliferation, migration and invasion were determined in U87 cells transfected with miR-365 mimic or miR-NC. ${ }^{* *} \mathrm{P}<0.01$.

upregulated in glioma tissues compared with adjacent normal tissues (Fig. 4A). In addition, Spearman's correlation analysis showed a reversed correlation between miR-365 expression levels and PIK3R3 mRNA levels in glioma tissues $(r=-0.563$, $\mathrm{P}=0.0002$; Fig. 4B).

Overexpression of PIK3R3 rescues the inhibition effect of miR-365 in glioma. To further illustrate whether miR-365 affects human glioma cell proliferation, migration and invasion through PIK3R3, U87 cells were co-transfected with
miR-365 or miR-NC and the overexpression PIK3R3 plasmid. Western blot analysis showed that miR-365 overexpression significantly decreased PIK3R3 protein expression, while overexpression PIK3R3 plasmid restored PIK3R3 expression (Fig. 5A). In addition, we found that PIK3R3 overexpression was able to counteract the inhibitory effect on cell proliferation (Fig. 5B), migration (Fig. 5C), and cell invasion (Fig. 5D) in glioma cells induced by miR-365 overexpression. These data indicated that miR-365 exerts suppressive role in glioma by repressing PIK3R3. 
A

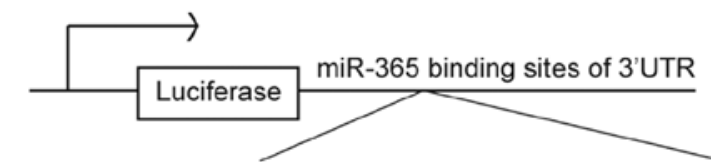

Wt PIK3R3 3'UTR 5'...UUAGACUACGAUGAGGGCAUUC...3

$\operatorname{miR}-365$

3'...UAUUCCUAAAAAUCCCCGUAAU...5'

Mut PIK3R3 3'UTR 5'...UUAGACUACGAUGAGCCCUUAC...3'
C

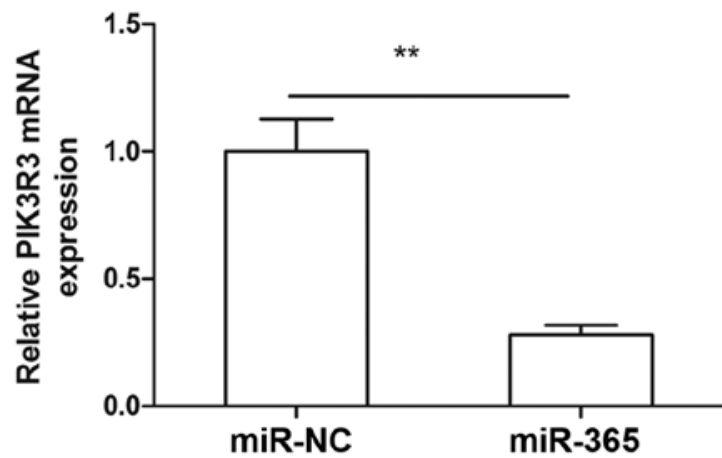

B
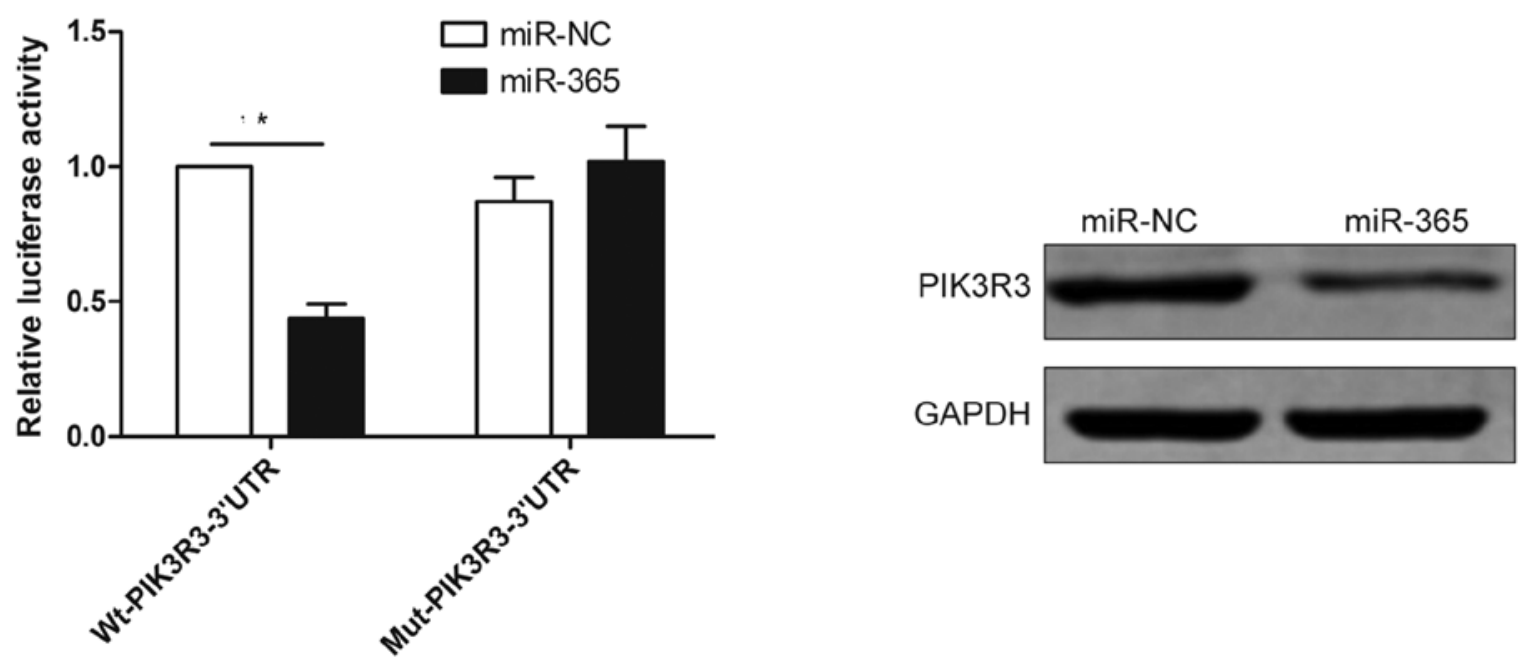

Figure 3. PIK3R3 is a candidate target of miR-365 in glioma cells. (A) Schematic construction of wild-type (Wt) and mutant (Mut) 3'-UTR of PIK3R3 according to miR-365 and its putative binding sequence in the 3'-UTR of PIK3R3 (position 67-73). (B) The luciferase activities were determined in U87 cells co-transfected with wild-type (Wt) or mutant-type (Mut) 3'-UTR of PIK3R3 reporter plasmid and miR-365 mimic or miR-NC. (C and D) The PIK3R3 mRNA expression and protein expression levels were determined in U87 cells transfected with miR-365 mimic or miR-NC by qRT-PCR and western blotting, respectively. GAPDH was used as a control. ${ }^{* *} \mathrm{P}<0.01$.

A

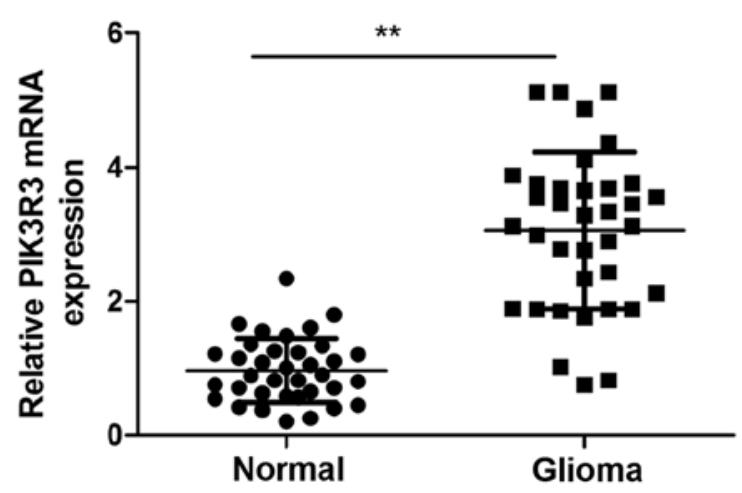

B

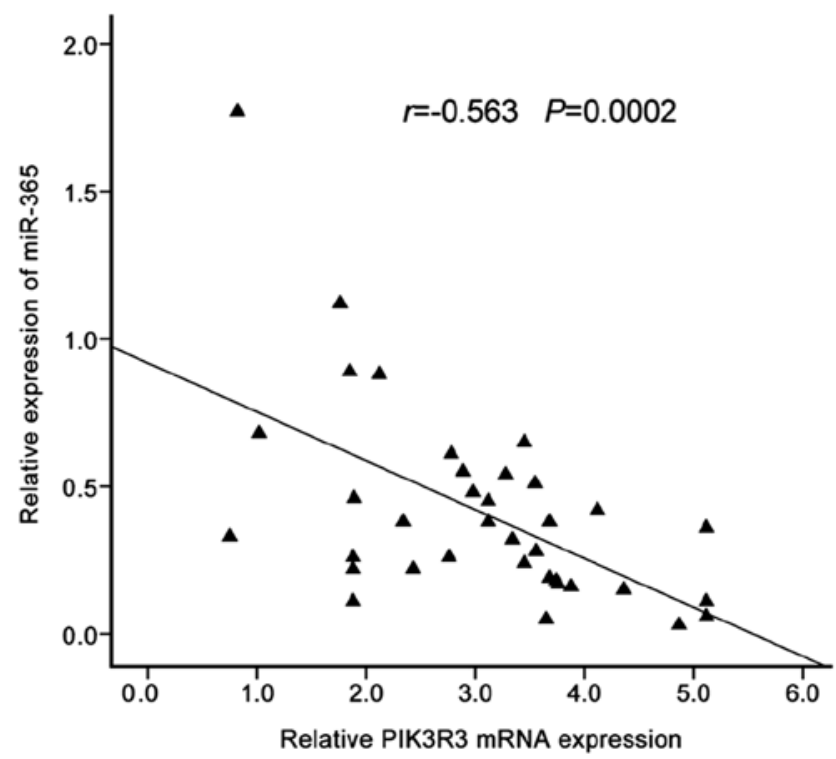

Figure 4. PIK3R3 mRNA expression level was upregulated in glioma tissues and inversely correlated with miR-365 expression. (A) PIK3R3 mRNA expression was detected in 36 paired glioma tissues and adjacent normal tissues by qRT-PCR. GAPDH was used as an internal control. (B) The inverse correlation between miR-365 and PIK3R3 was analyzed in clinical glioma samples by Spearman's correlation coefficient $(\mathrm{n}=36){ }^{* *} \mathrm{P}<0.01$. 
A

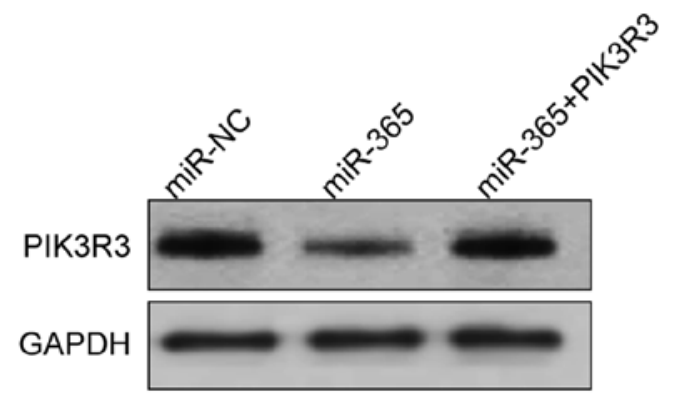

C

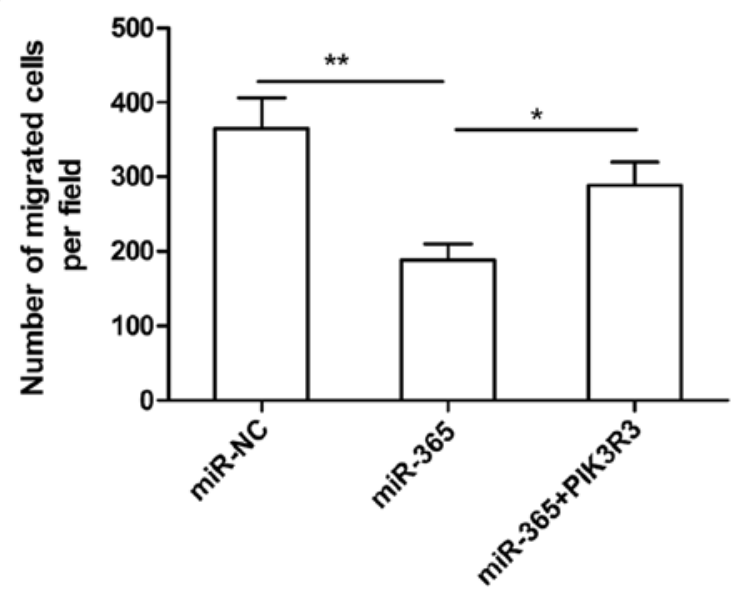

B

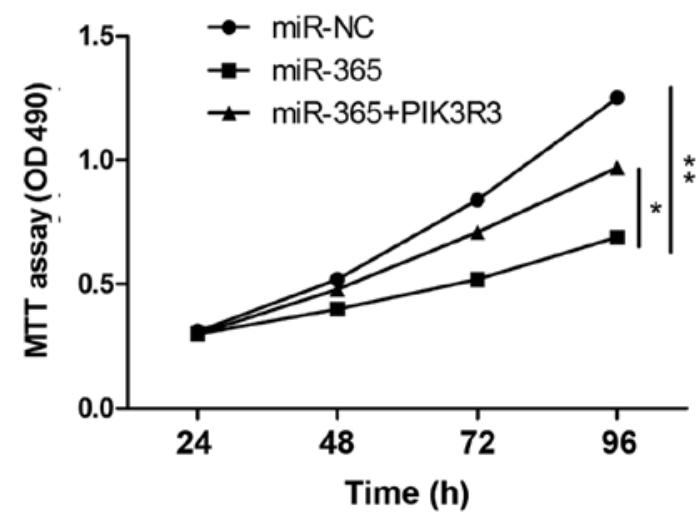

D

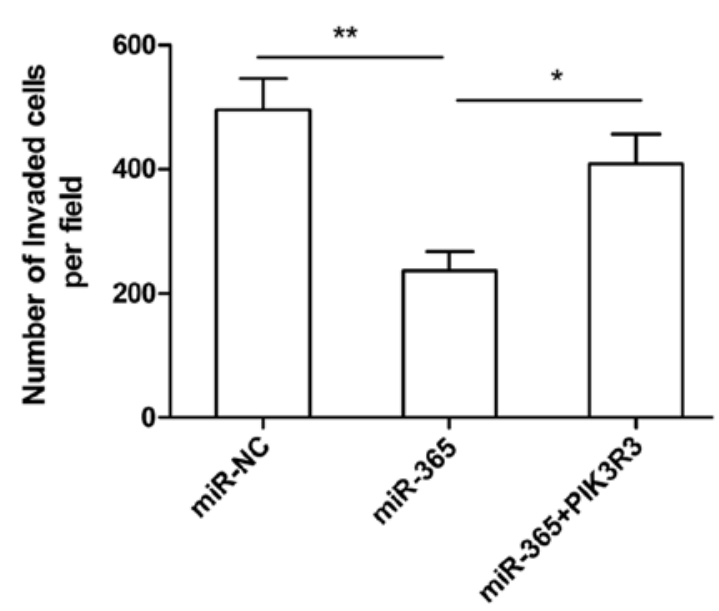

Figure 5. Overexpression of PIK3R3 reverses the suppressive effect of miR-365 in glioma. (A) PIK3R3 protein expression was determined in U87 cells transfected with miR-365 with/without PIK3R3 overexpression plasmid. GAPDH was used as the internal control. (B-D) Cell proliferation, migration and invasion were determined in U87 cells transfected with miR-365 with/without PIK3R3 overexpression plasmid. ${ }^{*} \mathrm{P}<0.05,{ }^{* *} \mathrm{P}<0.01$.

Upregulation of miR-365 suppresses tumor growth in mice. To investigate the role of miR-365 in tumor growth in vivo, U87 cells $\left(2 \times 10^{6}\right)$, stable overexpression of miR-365 mimic or miR-NC were injected subcutaneously into nude mice and the mice were monitored closely for tumor growth. As shown in Fig. 6A, tumor growth was slower in miR-365 overexpression group than that of miR-NC overexpression (Fig. 6A). At 35 days after injection, mice was sacrified and tumor tissues were excised and weighed. We found that tumor weight was decreased in miR-365 overexpression group compared to miR-NC overexpression group (Fig. 6B). In addition, miR-365 and PIK3R3 expression were determined in tumor xenograft tumors by qRT-PCR and western blotting, respectively. Compared to miR-NC overexpression group, miR-365 expression was increased (Fig. 6C), and PIK3R3 protein expression was decreased in miR-365 overexpression group (Fig. 6D). These results suggested that miR-365 suppressed glioma growth in vivo by repressing PIK3R3.

miR-365 regulates the AKT/mTOR signaling pathway. PIK3R3 has been showed to be involved in tumor progression by regulating AKT/mTOR signal pathway (21-23). Since PIK3R3 was confirmed as a target of miR-365 in the above results, we investigated whether miR-365 could regulate the AKT/mTOR pathway. We detected AKT, p-AKT, mTOR and p-mTOR protein expression in glioma cells and xenograft tumor tissues from nude mice. We found that miR-365 overexpression significantly inhibited phosphorylation of AKT and mTOR in glioma cells and xenograft tumor tissues. Total AKT and mTOR protein levels did not change. These data might indicate that miR-365 inhibit cell proliferation and invasion of glioma through indirectly regulating the AKT/mTOR signaling pathway (Fig. 7).

\section{Discussion}

Recently number of miRNAs have been identified to function as a tumor suppressor or an oncogene in glioma by regulating their target molecule $(11,12)$. For example, Zhu et al reported that miR-217 inhibited proliferation, colony formation, migration and invasion of glioma cells by repressing Runx2 (20). Chen et al found that overexpression of miR-19a by a miR-19a mimic promoted glioma cell proliferation and invasion by targeting the Ras homolog family member B (RhoB) (24). Peng et al also found that miR-506 was downregulated in glioma tissues and cell lines, and functions as a novel tumor suppressor to inhibit the proliferation, colony formation, migration and invasion of glioma cells in vitro, and suppress glioma tumor growth in vivo by targeting STAT3 (25). In the present study, our results revealed that miR-365 expression was significantly downregulated in glioma tissues 
A

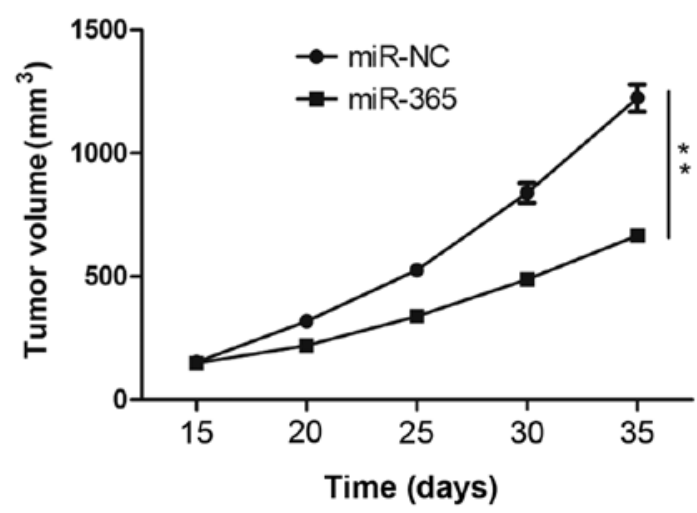

C

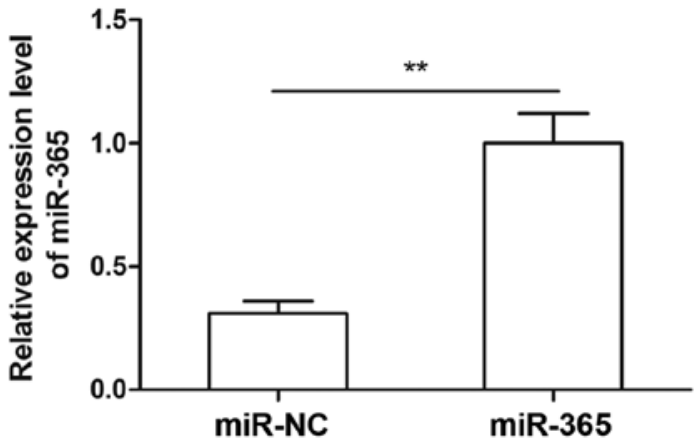

B

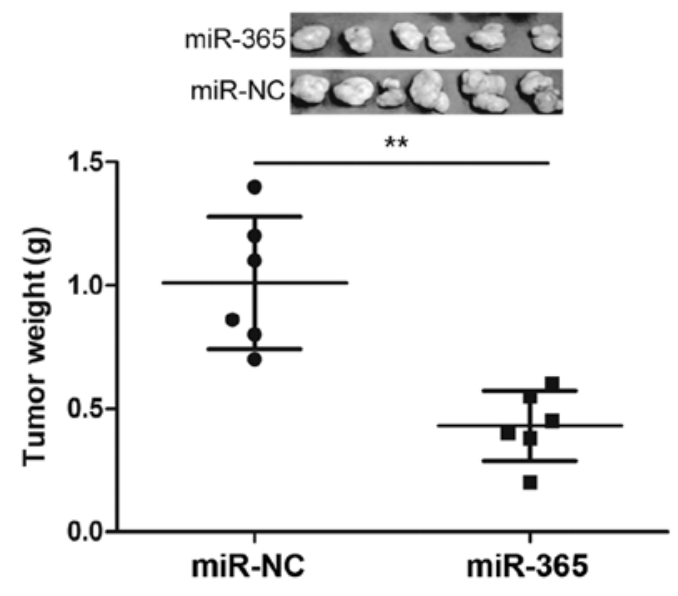

D

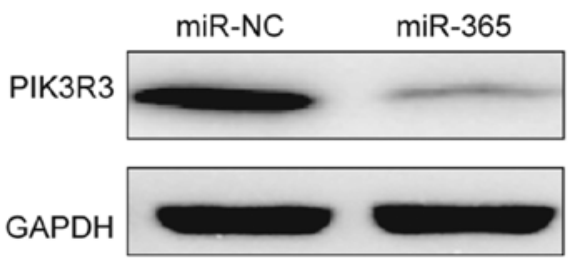

Figure 6. miR-365 suppresses tumor growth in vivo by repressing PIK3R3. (A) Growth curves for tumor volumes in xenografts of nude mice from different groups. (B) Tumor image and weight from different group. (C) miR-365 expression was determined in tumor tissues from different groups by qRT-PCR. (D) PIK3R3 protein level was determined in tumor tissues from different groups by western blotting. The GAPDH was used as an internal control. ${ }^{* *} \mathrm{P}<0.01$.

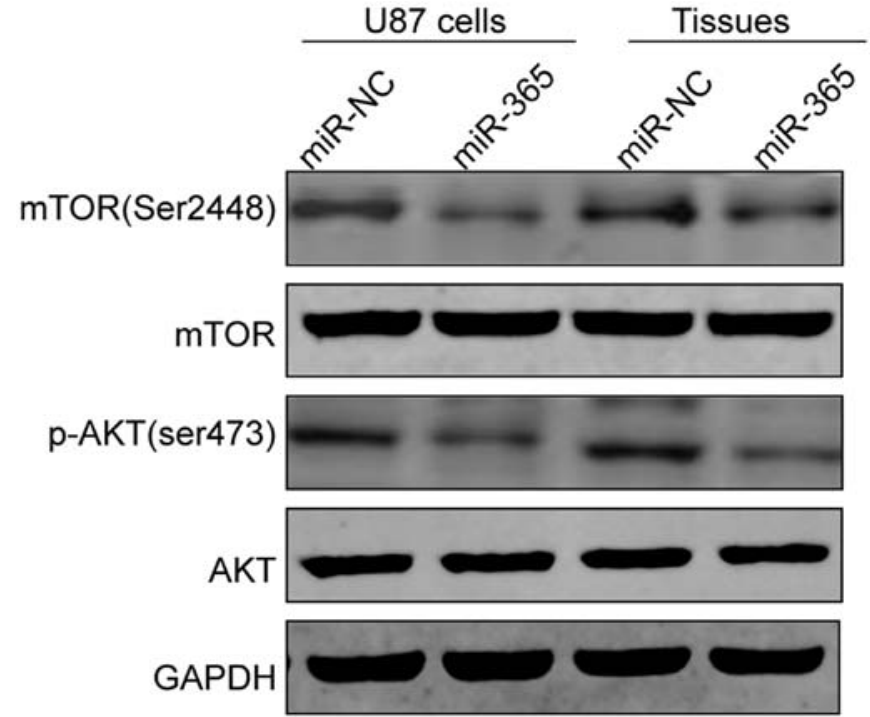

Figure 7. miR-365 regulates AKT/mTOR signaling pathway. AKT, p-AKT, mTOR and p-mTOR protein expression were determined in U87 cells transfected with miR-365 or miR-NC and xenograft tumor tissues from nude mice by western blotting. GAPDH was used as an internal control. ${ }^{* *} \mathrm{P}<0.01$. and cell lines compared to adjacent normal tissues and the normal cell line. Our results also demonstrated that miR-365 overexpression significantly decreased cell proliferation, migration and invasion of glioma in vitro, and suppressed tumor growth in vivo. These data suggest that miR-365 may play crucial role in glioma development.

Aberrant expression of miR-365 has been found in various human cancers. In gastric cancer (16), cutaneous squamous cell carcinoma (26) and pancreatic cancer (27), miR-365 expression is frequent upregulated and acts as an oncogene. On the contrary, in lung cancer $(13,14)$, melanoma $(15)$, osteosarcoma (17), and colon cancer (18), miR-365 expression was downregulated and functions as a tumor suppressor. However, the function and relevant mechanisms of miR-365 in glioma remains unclear. Herein, we found that miR-365 expression was significantly downregulated in glioma tissues and cell lines, and that miR-365 significantly inhibited cell proliferation, invasion and migration of glioma cells, and suppressed tumor growth in nude mice. These results suggested that miR-365 might function as a tumor suppressor in glioma.

PIK3R3 (phosphoinositide-3-kinase regulatory subunit 3), a member of the phosphatidylinositol 3-kinase (PI3K) family, 
has been suggested to play crucial roles in diverse biological processes, such as cell proliferation, differentiation, carcinogenesis and tumor angiogenesis $(28,29)$. Accumulating evidence suggested that PIK3R 3 was involved in tumor development and progression, and functions as an oncogene in multiple cancers, including ovarian cancer (22), gastric cancer (30), hepatocellular carcinoma (21), lung cancer (22), colorectal cancer (31), and breast cancer (28). It has been shown that PIK3R3 could regulate the AKT/mTOR pathway, which contribute to promotion of cancer progression (21-23). In glioma, it has been found that overexpression of PIK3R3 can promote growth of glioma cells in vitro (32), which imply PIK3R3 as an oncogene in glioma. In this study, PIK3R3 was proved to be a direct target of miR-365 in glioma cells, and its mRNA expression was inversely correlated with miR-365 expression in clinical glioma tissues. Overexpression of PIK3R3 in U87 cells reversed the inhibition effected on proliferation, migration and invasion induced by miR-365 overexpression. Of note, miR-365 was able to regulate PIK3R3 and its downstream protein p-AKT, p-mTOR expression in vitro and in vivo, which are key participants in the AKT/mTOR pathway. These findings might imply that miR-365 exerts it tumor-suppressing functions in glioma by regulating the PIK3R3/AKT/ mTOR signal pathway.

Our study provides evidence that miR-365 expression was downregulated in glioma tissues and cell lines, and that miR-365 suppressed glioma cell proliferation, migration, and invasion in vitro, as well as glioma growth in vivo by directly targeting PIK3R 3 and indirectly regulating the AKT/mTOR pathway. These results suggest that miR-365 could serve as a potential novel target for future glioma therapy.

\section{References}

1. Reardon DA, Rich JN, Friedman HS and Bigner DD: Recent advances in the treatment of malignant astrocytoma. J Clin Oncol 24: 1253-1265, 2006.

2. Clarke J, Butowski $\mathrm{N}$ and Chang S: Recent advances in therapy for glioblastoma. Arch Neurol 67: 279-283, 2010.

3. Grauer OM, Wesseling P and Adema GJ: Immunotherapy of diffuse gliomas: Biological background, current status and future developments. Brain Pathol 19: 674-693, 2009.

4. Fabian MR, Sonenberg N and Filipowicz W: Regulation of mRNA translation and stability by microRNAs. Annu Rev Biochem 79: 351-379, 2010.

5. Guo H, Ingolia NT, Weissman JS and Bartel DP: Mammalian microRNAs predominantly act to decrease target mRNA levels. Nature 466: 835-840, 2010

6. Almeida MI, Reis RM and Calin GA: MicroRNA history: Discovery, recent applications, and next frontiers. Mutat Res 717: $1-8,2011$.

7. Bartel DP: MicroRNAs: Genomics, biogenesis, mechanism, and function. Cell 116: 281-297, 2004.

8. Lu J, Getz G, Miska EA, Alvarez-Saavedra E, Lamb J, Peck D, Sweet-Cordero A, Ebert BL, Mak RH, Ferrando AA, et al: MicroRNA expression profiles classify human cancers. Nature 435: 834-838, 2005.

9. Farazi TA, Spitzer JI, Morozov P and Tuschl T: miRNAs in human cancer. J Pathol 223: 102-115, 2011.

10. McManus MT: MicroRNAs and cancer. Semin Cancer Biol 13: 253-258, 2003.

11. England B, Huang T and Karsy M: Current understanding of the role and targeting of tumor suppressor p53 in glioblastoma multiforme. Tumour Biol 34: 2063-2074, 2013.

12. Tivnan A and McDonald KL: Current progress for the use of miRNAs in glioblastoma treatment. Mol Neurobiol 48: 757-768, 2013.
13. Qi J, Rice SJ, Salzberg AC, Runkle EA, Liao J, Zander DS and Mu D: MiR-365 regulates lung cancer and developmental gene thyroid transcription factor 1. Cell Cycle 11: 177-186, 2012.

14. Sun R, Liu Z, Ma G, Lv W, Zhao X, Lei G and Xu C: Associations of deregulation of mir-365 and its target mRNA TTF-1 and survival in patients with NSCLC. Int J Clin Exp Pathol 8: 2392-2399, 2015.

15. Bai J, Zhang Z, Li X and Liu H: MicroRNA-365 inhibits growth, invasion and metastasis of malignant melanoma by targeting NRP1 expression. Int J Clin Exp Pathol 8: 4913-4922, 2015.

16. Guo SL, Ye H, Teng Y, Wang YL, Yang G, Li XB, Zhang C, Yang X, Yang ZZ and Yang X: Akt-p53-miR-365-cyclin D1/ cdc25A axis contributes to gastric tumorigenesis induced by PTEN deficiency. Nat Commun 4: 2544, 2013.

17. Gao J, Zhao P, Chen X, Wang W, Li Y, Xi W, Zhang W, Hu P, Wang T and Shan L: miR-365 inhibits proliferation and promotes apoptosis of SOSP9607 osteosarcoma cells. Xi Bao Yu Fen Zi Mian Yi Xue Za Zhi 32: 44-48, 2016 (In Chinese).

18. Nie J, Liu L, Zheng W, Chen L, Wu X, Xu Y, Du X and Han W: microRNA-365, down-regulated in colon cancer, inhibits cell cycle progression and promotes apoptosis of colon cancer cells by probably targeting Cyclin D1 and Bcl-2. Carcinogenesis 33: 220-225, 2012.

19. Liu K, Li X, Cao Y, Ge Y, Wang J and Shi B: MiR-132 inhibits cell proliferation, invasion and migration of hepatocellular carcinoma by targeting PIK3R3. Int J Oncol 47: 1585-1593, 2015.

20. Zhu Y, Zhao H, Feng L and Xu S: MicroRNA-217 inhibits cell proliferation and invasion by targeting Runx 2 in human glioma. Am J Transl Res 8: 1482-1491, 2016.

21. Cao G, Dong W, Meng X, Liu H,Liao Hand Liu S: MiR-511 inhibits growth and metastasis of human hepatocellular carcinoma cells by targeting PIK3R3. Tumour Biol 36: 4453-4459, 2015.

22. Xu L, Wen Z, Zhou Y, Liu Z, Li Q, Fei G, Luo J and Ren T: MicroRNA-7-regulated TLR9 signaling-enhanced growth and metastatic potential of human lung cancer cells by altering the phosphoinositide-3-kinase, regulatory subunit 3/Akt pathway. Mol Biol Cell 24: 42-55, 2013.

23. Zhang L, Huang J, Yang N, Greshock J, Liang S, Hasegawa K, Giannakakis A, Poulos N, O'Brien-Jenkins A, Katsaros D, et al: Integrative genomic analysis of phosphatidylinositol 3'-kinase family identifies PIK3R3 as a potential therapeutic target in epithelial ovarian cancer. Clin Cancer Res 13: 5314-5321, 2007.

24. Chen Q, Guo W, Zhang Y, Wu Y and Xiang J: MiR-19a promotes cell proliferation and invasion by targeting RhoB in human glioma cells. Neurosci Lett 628: 161-166, 2016 .

25. Peng T, Zhou L, Zuo L and Luan Y: MiR-506 functions as a tumor suppressor in glioma by targeting STAT3. Oncol Rep 35: 1057-1064, 2016.

26. Zhou M, Liu W, Ma S, Cao H, Peng X, Guo L, Zhou X, Zheng L, Guo L, Wan M, et al: A novel onco-miR-365 induces cutaneous squamous cell carcinoma. Carcinogenesis 34: 1653-1659, 2013.

27. Hamada S, Masamune A, Miura S, Satoh K and Shimosegawa T: MiR-365 induces gemcitabine resistance in pancreatic cancer cells by targeting the adaptor protein SHC1 and pro-apoptotic regulator BAX. Cell Signal 26: 179-185, 2014.

28. Klahan S, Wu MS, Hsi E, Huang CC, Hou MF and Chang WC: Computational analysis of mRNA expression profiles identifies the ITG family and PIK3R3 as crucial genes for regulating triple negative breast cancer cell migration. BioMed Res Int 2014: 536591, 2014

29. Xia X, Cheng A, Akinmade D and Hamburger AW: The $\mathrm{N}$-terminal 24 amino acids of the p55 gamma regulatory subunit of phosphoinositide 3-kinase binds $\mathrm{Rb}$ and induces cell cycle arrest. Mol Cell Biol 23: 1717-1725, 2003.

30. Zhou J, Chen GB, Tang YC, Sinha RA, Wu Y, Yap CS, Wang G, $\mathrm{Hu} \mathrm{J}, \mathrm{Xia} \mathrm{X}$, Tan P, et al: Genetic and bioinformatic analyses of the expression and function of PI3K regulatory subunit PIK3R3 in an Asian patient gastric cancer library. BMC Med Genomics 5: 34, 2012.

31. Wang G, Yang X, Li C, Cao X, Luo X and Hu J: PIK3R3 induces epithelial-to-mesenchymal transition and promotes metastasis in colorectal cancer. Mol Cancer Ther 13: 1837-1847, 2014

32. Soroceanu L, Kharbanda S, Chen R, Soriano RH, Aldape K, Misra A, Zha J, Forrest WF, Nigro JM, Modrusan Z, et al: Identification of IGF2 signaling through phosphoinositide3-kinase regulatory subunit 3 as a growth-promoting axis in glioblastoma. Proc Natl Acad Sci USA 104: 3466-3471, 2007. 